\title{
The track-based alignment of the ALFA Roman Pot detectors of the ATLAS experiment
}

\author{
Krzysztof Janas* on behalf of the ATLAS Collaboration \\ AGH University of Science and Technology, Kraków, Poland \\ E-mail: Janaslagh.edu.pl
}

\begin{abstract}
ALFA is part of the ATLAS Roman Pot detector dedicated to measure protons scattered at very small angles in proton-proton collisions at the LHC. ALFA aims to study elastic and diffractive events in special runs with reduced luminosity and optimized beam optics. Precision of the measurement depends on the correct positioning of the ALFA detector with respect to the actual beam position. For this purpose a track-based procedure is used which utilizes tracks of beam halo and elastic protons collected during physics data taking. This paper presents results of fast and robust alignment of ALFA detectors during LHC Run 2. The alignment constants are used in analysis of diffractive events and also serve as preliminary values in elastic event analysis. Precise measurements of the elastic cross sections require more precise dedicated alignment.
\end{abstract}

7th Annual Conference on Large Hadron Collider Physics - LHCP2019

20-25 May, 2019

Puebla, Mexico

${ }^{*}$ Speaker. 


\section{ALFA Detector}

The ALFA detector (Absolute Luminosity For ATLAS) is a high-precision scintillating fibers detector that is designed to measure protons scattered at very small angle. The ATLAS tracking system covers the pseudorapidity range $|\eta|<2.5$ and the calorimetric measurements range up to $|\eta|=4.9$ [1]. To obtain acceptance with $|\eta|>8.5$, tracking stations are placed at distance about 240 $\mathrm{m}$ from the ATLAS Interaction Point (IP) and housed in so-called Roman Pots (RP), that can be moved very close to the beam [2]. The detector was designed for absolute luminosity measurements by studying the properties of the elastic scattering, but data collected with it can also be used to analyze the diffractive processes.

The ALFA detector has two stations on each side of the central ATLAS detector - Fig. 1. Two stations named B7L1 and A7L1 are placed on side A; and two stations: A7R1 and B7R1 are placed on the side C. Each station is composed of movable upper and lower RP, with one Main Detector (MD) for tracking protons and two Overlap Detectors (OD) for distance measurement. They are completed by trigger scintillators covering the active areas of MDs and ODs - Fig. 2.

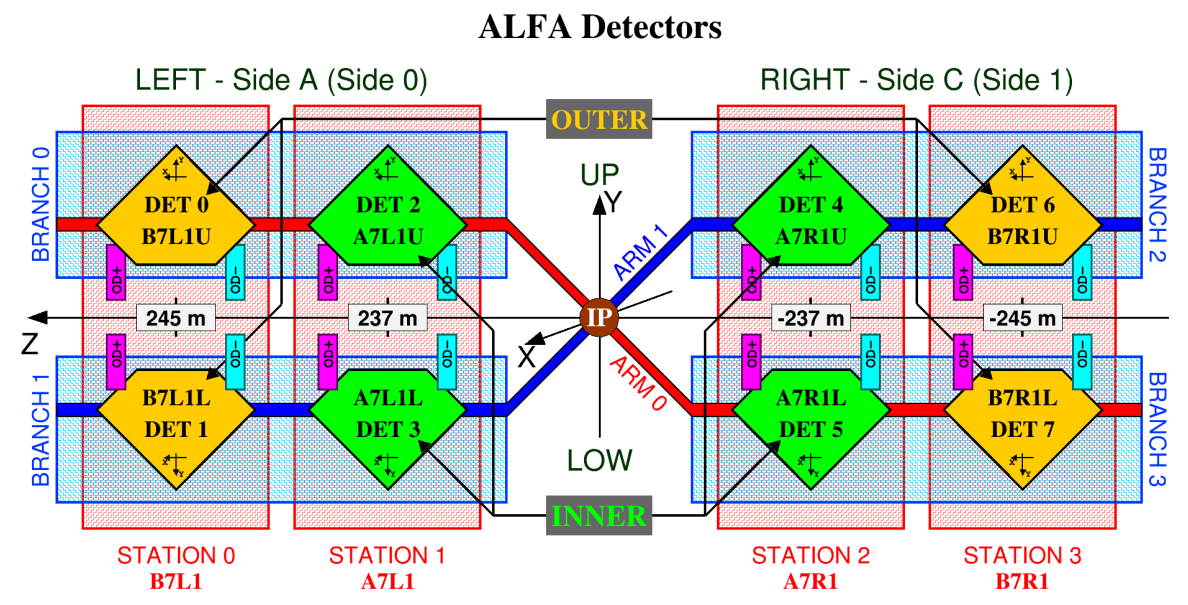

Figure 1: A sketch of the experimental set-up, showing the naming scheme of the ALFA detectors around the ATLAS IP. The detectors can be grouped into two arms for elastic-scattering event topologies.

The aim of the MD is to track the protons scattered at small angle. It is used for physical data collection. Each MD consists of 2 sets of 10 layers with 64 square scintillating fibers with $0.5 \mathrm{~mm}$ side length glued on both sides of titanium plates, arranged at angles of $\pm 45^{\circ}$ with respect to the $\mathrm{y}$-axis. In each $\mathrm{MD}$, the layers are arranged perpendicular to the beam direction. The local tracks ( $\mathrm{X}$ and $\mathrm{Y}$ coordinates) are reconstructed from the hit pattern of protons traversing the scintillating fibers.

Two independent ODs are attached at each side of both MDs, as sketched in Fig. 2. The ODs from upper and lower RPs are overlapping, which is used for tracking the same particles in both OD and measuring the distance between them. The ODs are built of the same scintillating fibers as MD, but there are only 3 layers of 30 fibers each and the ODs can measure only the vertical position of the track. 


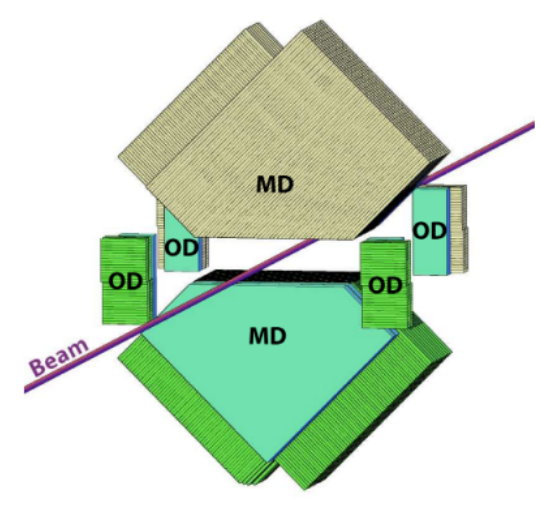

Figure 2: A schematic view of the ALFA station, composed of upper and lower RPs [2]. Each RP contains one Main Detector (MD) and two Overlap Detectors (OD). The plain objects visible in front of the lower MD and upper ODs are the trigger counters (for upper MD and the lower ODs they are on the opposite side).

A concept of the RP can be seen in Fig. 3, where the retracted and working positions are shown. The working position will bring the bottom surface of a pot to a minimal distance of few $\sigma$ from the circulating beam, but it can only happen during the dedicated high $\beta^{*}$ runs [3]. The mobility of RPs is one of the main reasons why precise alignment is so important.

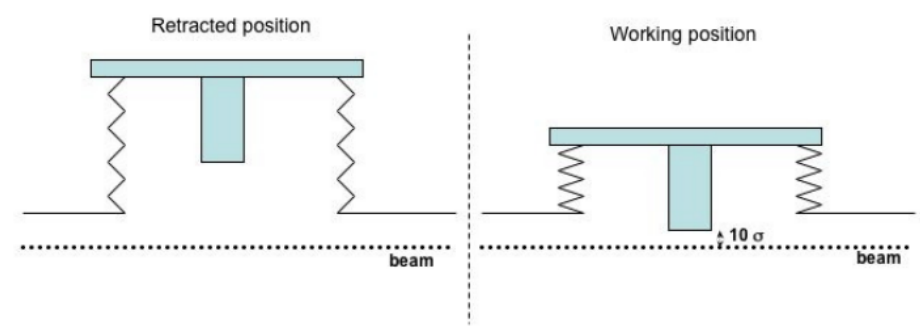

Figure 3: RP concept: on the left the retracted position is shown where the Pot is placed out from the beam; on the right in working position, the Pot is approached $10 \sigma$ from the circulating beam [3].

\section{Fast ALFA Alignment}

To allow correct reconstruction of scattering angle at IP the detectors must be aligned with respect to the beams. Therefore the alignment procedure must be repeated for each run to avoid bias due to possible beam movements.

Each detector has three degrees of freedom in the XY plane as indicated in Fig. 4:

- $\Delta X$ - horizontal position;

- $\Delta Y$ - vertical position;

- $\theta_{Z}$ - rotation along the $\mathrm{Z}$ axis. 
Other possible degrees of freedom $\left(\Delta Z, \theta_{X}, \theta_{Y}\right)$ are irrelevant, as they do not have significant impact to measuring the $\mathrm{X}$ and $\mathrm{Y}$ coordinates.

The vertical position can be calculated in two steps for both detectors in one ALFA station:

I. Distance between the upper and the lower detector (the first part of ALFA alignment using ODs analysis).

II. Vertical offset of the whole station (the second part of ALFA alignment using track-based analysis, parallel with calculation of the horizontal offset and rotation).

The distance measurement is the first stage of ALFA alignment using beam halo protons that travel parallel to the circulating beam and are reconstructed by ODs. The second stage is based on symmetry of elastic event patterns - an ellipse elongated in the vertical direction. Elastic selection criteria are based on the position of the tracks and thus depend on the alignment. In order to reach a convergence, the procedure is repeated several times. Offsets in $\mathrm{X}$ and $\mathrm{Y}$ and rotation are calculated in each iteration, and the corrections are used in the next iteration.

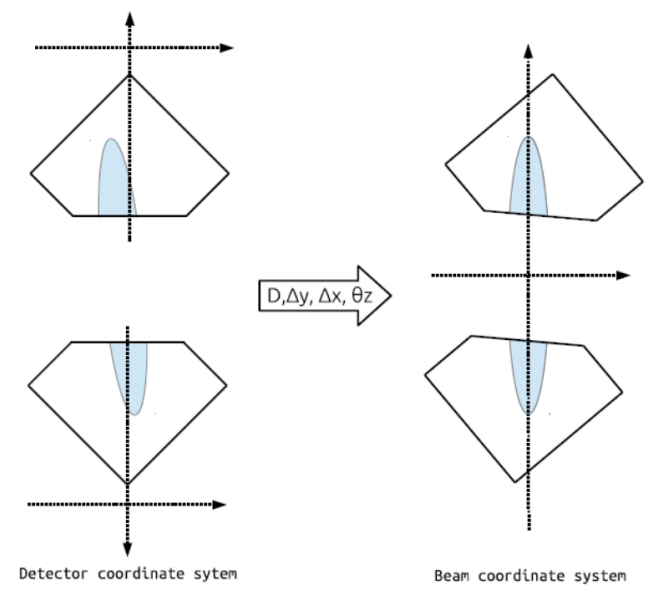

Figure 4: Principle of the ALFA alignment [4], based on four parameters: distance between the detectors, vertical offset of the whole station, horizontal offset and rotation angle of the detector. In beam coordinate system, the elastic pattern of tracks is symmetrical. This property is used to align detectors.

\section{OD Based Alignment}

The RPs are inserted only for special runs and the distance needs to be measured each time. The OD is designed to measure the vertical coordinate of traversing beam-halo particles or shower fragments. Halo particles originate from beam particles which left the bunch structure of the beam but still circulate in the beam pipe. These events can be used to determine the distance between upper and lower RPs as shown in Fig. 5.

The halo particles are mostly parallel to the beam and hit fibers in upper and lower OD in the ALFA station with the same vertical position. The measurement is done separately for left and right OD sides and then averaged. 


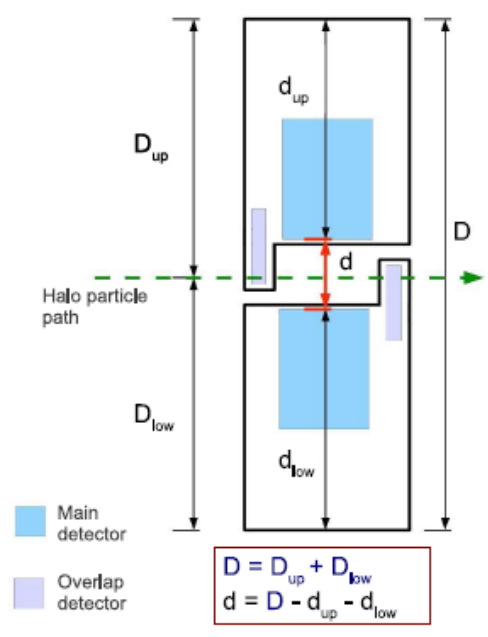

Figure 5: Scheme of the Overlap Detectors concept: halo particle hit fibers in upper and lower OD in the ALFA station with the same vertical position [2]. The measured positions can be used to determine the distance $\mathbf{d}$ between upper and lower MD.

Additional detectors that measure the positions of the RPs are Linear Variable Displacement Transducers (LVDT) which are calibrated in a laser survey. This measurement is used during data collection, for a preliminary set of distances between ALFA detectors. It can be also used to cross check the results from OD analysis. Correlation between the Fast ALFA Alignment results and the LVDT measurement for station A7R1 for the diffractive runs from October 2015 ALFA campaign is presented in Fig. 6.

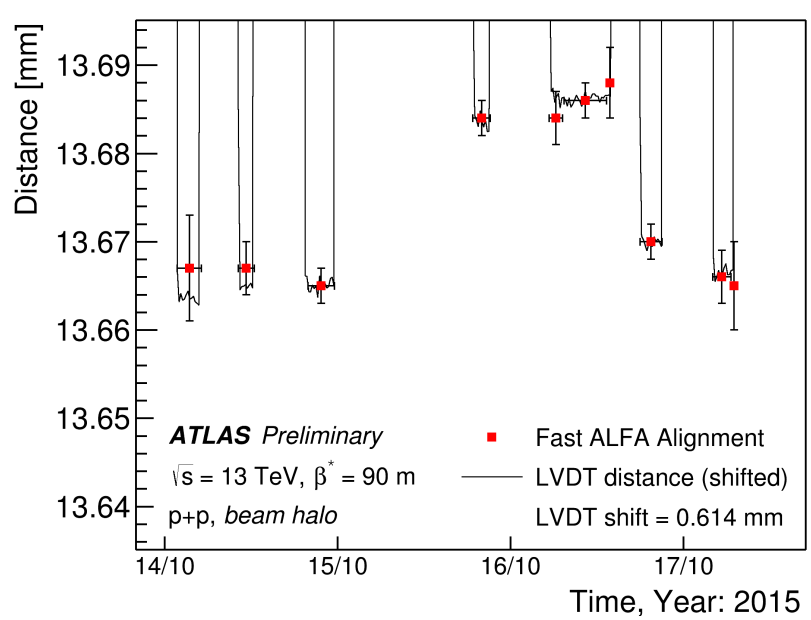

Figure 6: Correlation between the Fast ALFA Alignment results and the LVDT measurement for station A7R1 for the diffractive runs from October 2015 ALFA campaign. Vertical error bars show the statistical uncertainty while the horizontal bars correspond to the run length. The LVDT results are shifted by $0.614 \mathrm{~mm}$ to match the Fast ALFA Alignment results (for easier comparison). 


\section{MD Based Alignment}

MD Based Alignment is performed using tracks of protons scattered in elastic interactions and reconstructed with MD. In the beam coordinate system, the elastic pattern of tracks is symmetrical, what can be seen in Fig. 4. The left-right symmetry is used to obtain the $X$ offset, up-down symmetry provides the $\mathrm{Y}$ offset, and the means of the correlation between the horizontal and vertical positions of the reconstructed tracks determine the rotation of the detector.

In Figures 7-9 position distributions for the detector B7L1U and station B7L1 for LHC fill 4505 are shown after selection of elastic events is imposed. The plots are obtained in the last iteration of the alignment procedure. They are used to determine the alignment parameters:

- The center of the distribution of horizontal position of elastic protons is used for X offset estimation - Fig. 7. The center is calculated using three different methods: arithmetic mean (default), median value and Gaussian mean.

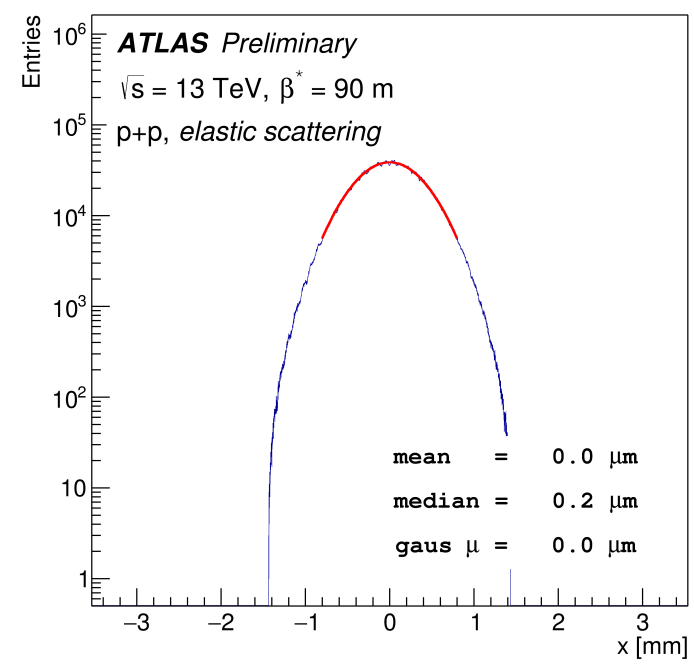

Figure 7: Distribution of horizontal positions of elastic protons measured in detector B7L1U that is used for X offset estimation.

- The detectors are aligned if both distributions of vertical positions of elastic protons are overlapping in the selected range. For this, one of them is shifted left and the other right by the same amount so as to achieve a constant ratio between distributions, because slope indicates misaligned detectors - Fig. 8. Convolution with the Gaussian function is applied to smooth the distributions. The shift is a measure of the $\mathrm{Y}$ offset between the center of the ALFA station with respect to the actual beam position.

- The slope of linear fit to the correlations between the vertical position and average horizontal position indicates the rotation angle $\theta_{Z}$ of the detector - Fig. 9. Two methods are used to determine the center of the horizontal distribution for each of the vertical bins: arithmetic mean (red) and Gaussian mean (green). 


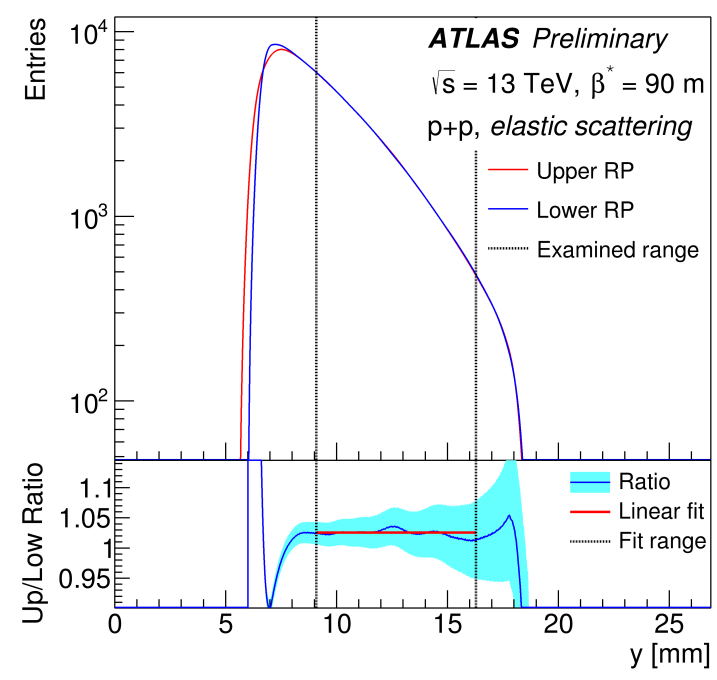

Figure 8: Distributions of vertical positions of elastic protons measured in station B7L1 that are used for $\mathrm{Y}$ offset estimation. The ratio between distributions is shown with statistical uncertainties. The red line shows a linear fit. A slope equal to zero indicates aligned detectors.

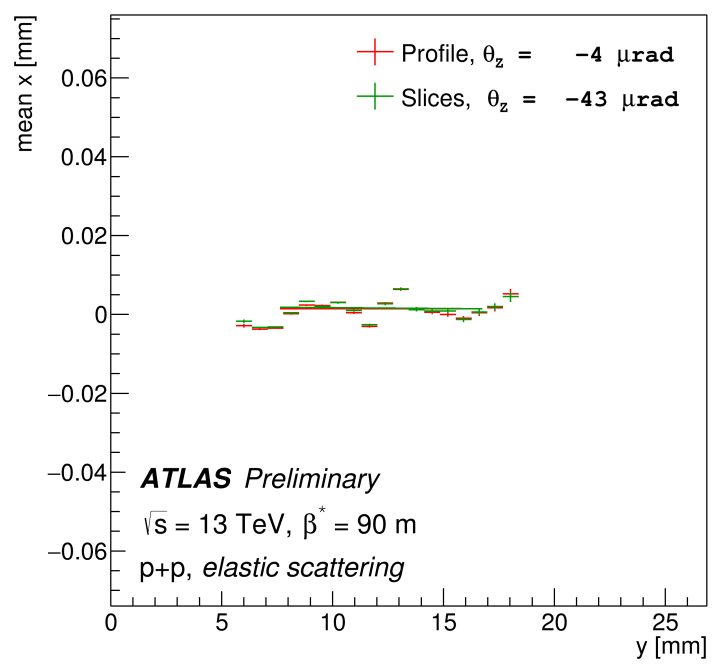

Figure 9: Correlation between the vertical positions and average horizontal positions of elastic protons measured in detector B7L1U that is used for rotation angle estimation. The lines show linear fits that are used for estimation of the rotation angle around the middle of the lower $\mathrm{Y}$ detector edge. 


\section{Alignment Parameters vs. Fill Number}

As mentioned earlier, the detectors must be aligned with respect to the beam. Therefore the alignment procedure must be repeated for each run to avoid bias due to possible beam movements. However, for different LHC fills during October 2015 ALFA campaign, the results of the alignment procedure tend to be similar. The relative values of alignment parameters are presented in Fig. 10. Some variations for $\mathrm{X}$ and $\mathrm{Y}$ offsets are visible, but the rotation parameter is constant within uncertainties. What is important is that the $\mathrm{X}$ and $\mathrm{Y}$ offsets vary in the same way for all detectors on one side. This fact implicates that the main reason for this variation is beam movement.

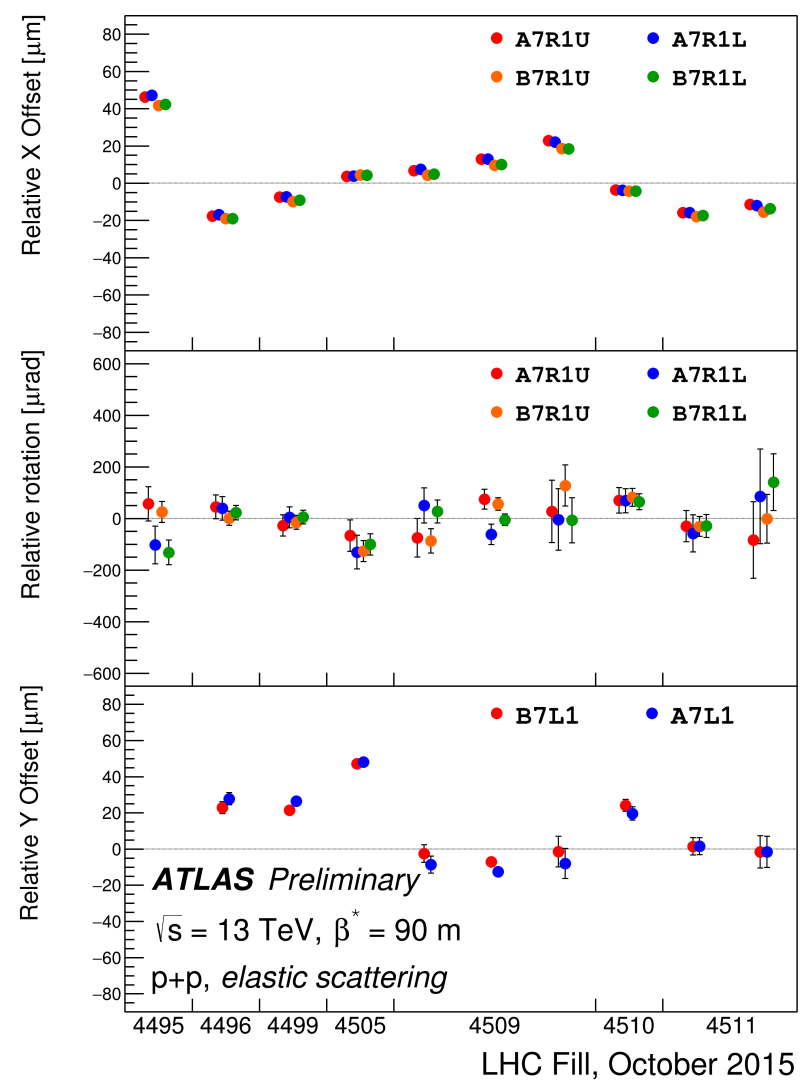

Figure 10: Alignment parameters (X offset, rotation, $\mathrm{Y}$ offset) for different LHC fills - for the detectors on the side A [5]. For easier comparison the median over all runs of each parameter is subtracted for each detector. Only statistical uncertainties are shown. Relative offsets in X and $\mathrm{Y}$ are very similar for all detectors. This suggests that the effect is related to the different beam position during each run. The fill dependence for rotation is negligible.

\section{Acknowledgments}

This work was supported in part by Polish National Science Center grant no. 2015/19/B/ST2/00989, by a joint project of the Polish National Agency for Academic Exchange and AGH UST no. PPI/PRO/2018/1/00026/U/001, and by PL-Grid infrastructure. 


\section{References}

[1] ATLAS collaboration, The ATLAS Experiment at the CERN Large Hadron Collider, JINST 3 (2008) S08003.

[2] ATLAS collaboration, Measurement of the total cross section from elastic scattering in pp collisions at $\sqrt{s}=7$ TeV with the ATLAS detector, Nucl. Phys. B889 (2014) 486 [1408. 5778].

[3] P. Jenni, M. Nordberg, M. Nessi and K. Jon-And, ATLAS Forward Detectors for Measurement of Elastic Scattering and Luminosity, Technical report, CERN, 2008-01-17.

[4] S. Jakobsen, Commissioning of the Absolute Luminosity For ATLAS detector at the LHC, Ph.D. thesis, CERN, 2013-12-16.

[5] ATLAS collaboration, Public Forward Detector Plots for Collision Data, https://twiki.cern.ch/twiki/bin/view/AtlasPublic/ForwardDetPublicResults. 